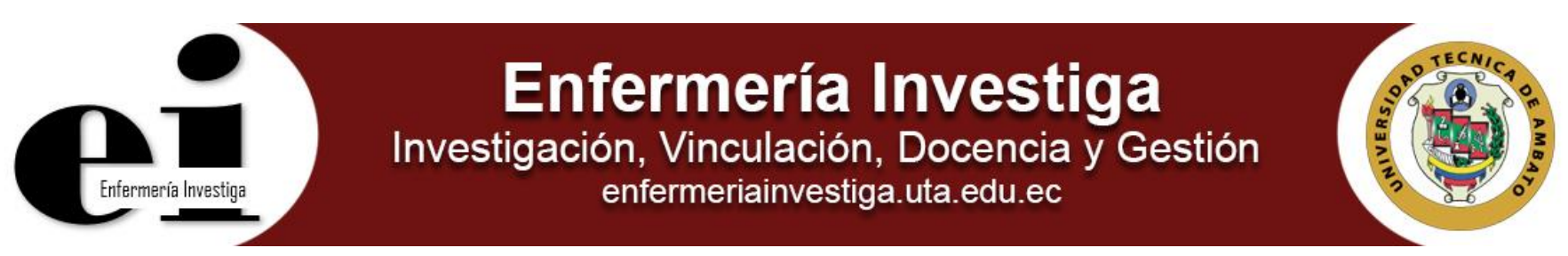

Presentación de casos

\title{
Glaucoma secundario a melanoma uveal
}

Glaucoma secondary to uveal melanoma

\author{
Nairovys Gómez Martínez¹, Nayaris Gómez Martínez², Roberto Primelles Hernandez²
}

${ }^{1}$ Universidad Regional Autónoma de los Andes - Facultad de Ciencias Médicas - Carrera de Enfermería - Ambato - Ecuador.

${ }^{2}$ Hospital Abel Santamaría Cuadrado - Servicio de Oftalmología - Pinar del Río - Cuba.

Gómez MN, Gómez MN, Primelles HR. Glaucoma secundario a melanoma uveal. Enferm Inv (Ambato). 2017; 2(3):115-117.

2477-9172 / 2550-6692 Derechos Reservados @ 2017 Universidad Técnica de Ambato, Carrera de Enfermería. Este es un artículo de acceso abierto distribuido bajo los términos de la Licencia Creative Commons, que permite uso ilimitado, distribución y reproducción en cualquier medio, siempre que la obra original es debidamente citada.

\section{Historia:}

Recibido: 23 junio 2017

Revisado: 19 julio 2017

Aceptado: 04 agosto 2017

Palabras Claves: Glaucoma; melanoma; úvea; iris

Keywords: Glaucoma; melanoma; uvea; iris

\section{Resumen}

Introducción: Los tumores localizados en iris y cuerpo ciliar son poco frecuentes y puede ser valorada su resección local como alternativa terapéutica. El melanoma uveal representa el 79$81 \%$ de los melanomas oculares y el $3-5 \%$ de todos los melanomas.

Objetivo: Dar a conocer el caso de una patología infrecuente con pocos casos reportados en la literatura, enfatizar su importancia dentro de los diagnósticos diferenciales y del estudio histopatológico.

Descripción del caso: Paciente femenina de 47 años de edad, quien acudió a la consulta de oftalmología, para valoración de una lesión pigmentada en endotelio corneal y en iris asociada a hipertensión ocular y pérdida de la agudeza visual en el ojo izquierdo de cuatro meses de evolución. La biomicroscopía ultrasónica confirmó la presencia de una masa iridiana, localizada en la periferia del iris. El estudio anatomopatológico mostró una proliferación de células névicas pleomórficas que reemplazaban el estroma normal del iris.

Conclusiones: En pacientes que presentan una clínica de glaucoma unilateral refractario a tratamiento farmacológico junto con dispersión de pigmento es importante una exploración exhaustiva destacando el papel de la biomicroscopía ultrasónica.

\begin{abstract}
Introduction: Located in iris and ciliary body tumors are rare and its local resection as a therapeutic alternative can be valued. Uveal melanoma represents $79-81 \%$ of ocular melanomas and $3-5 \%$ of all melanomas.

Objetive: Let know the case of a rare disease with few cases reported in the literature, emphasize its importance within the differential diagnosis and histopathological study.

Case description: Female patient of 47-year-old, who attended the consultation in ophthalmology, for estimation of a pigmented lesion on corneal endothelium and iris associated with ocular hypertension and loss of visual acuity in the left eye of four months. Ultrasonic biomicroscopy confirmed the presence of a mass iridis, located on the periphery of the iris. The histopathological study showed a proliferation of cell Nevus Pleomorphic that replaced the normal stroma of the iris.

Conclusions: In patients presenting a clinic of unilateral glaucoma refractory to drug therapy along with pigment dispersion is important an exploration thorough highlighting the role of ultrasonic biomicroscopy.
\end{abstract}




\section{Introducción}

El globo ocular tiene una túnica vascular denominada úvea, que está integrada por el iris, el cuerpo ciliar y la coroide. Los tumores localizados en iris y cuerpo ciliar son poco frecuentes y puede ser valorada su resección local como alternativa terapéutica ${ }^{1}$.

El melanoma uveal representa el $79-81 \%$ de los melanomas oculares y el 3-5\% de todos los melanomas. En los Estados Unidos, la incidencia de melanoma uveal es de cinco millones de habitantes. En Europa, la incidencia de melanoma uveal sigue una de norte a sur de gradiente decreciente desde dos a ocho millones de habitantes ${ }^{2}$. El melanoma de iris representa aproximadamente un $4 \%$ de los melanomas de úvea. Puede asociar glaucoma en un14 a $30 \%{ }^{3}$.

El propósito del presente estudio fue dar a conocer el caso de una patología infrecuente con pocos casos reportados en la literatura, enfatizar su importancia dentro de los diagnósticos diferenciales y del estudio histopatológico como método para obtener un diagnóstico definitivo y realizar un abordaje temprano.

\section{Descripción del caso}

Paciente femenina de 47 años de edad, ama de casa, de procedencia urbana, con antecedentes patológicos personales generales y oculares de salud anterior, quien acudió el 12 de agosto del año 2014 a la consulta de oftalmología, remitida desde su área de salud para valoración de una lesión pigmentada en endotelio corneal y en iris asociada a hipertensión ocular y pérdida de la agudeza visual en el ojo izquierdo (OI) de cuatro meses de evolución. En la exploración oftalmológica presentaba:

- Agudeza Visual sin corrección de 1.0 en su ojo derecho (OD) y en el OI 0.4 (por carta de Snellen).

- Agudeza visual corregida 1.0 en OD y 0.5 en OI.

- La presión intraocular (PIO) fue en el OD de 10 $\mathrm{mmHg}$ y en el OI de $40 \mathrm{mmHg}$.

- Biomicroscopia con lámpara de hendidura de polo anterior se observó una masa melanótica en periferia de iris y endotelio corneal entre las 3 a 5 horas (figura 1).

Figura 1. Biomicroscopía en lámpara de hendidura

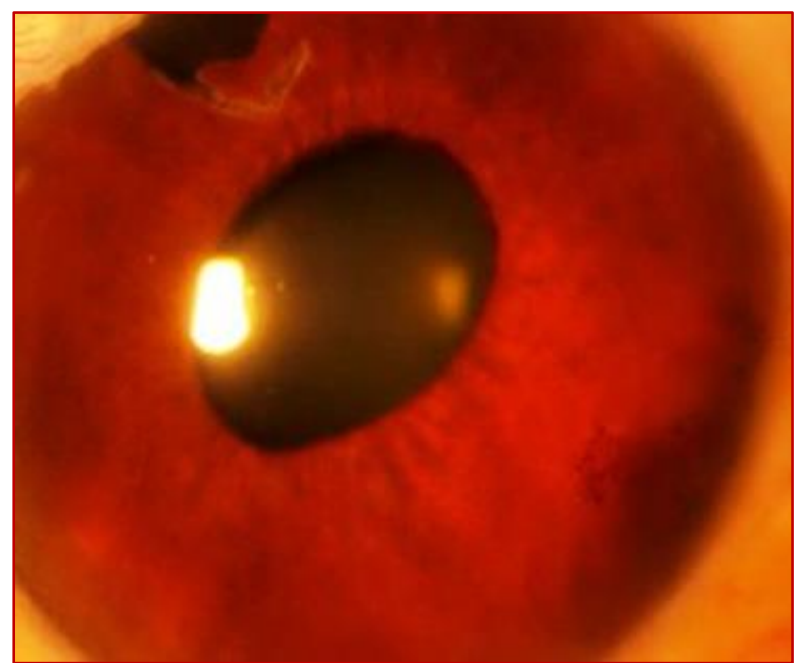

- $\quad$ Presión intraocular por tonómetro de Goldman OD $12 \mathrm{mmHg}$ y en Ol $40 \mathrm{mmHg}$.

- Gonioscopia se observó que la masa invadía el ángulo camerular y que la malla trabecular estaba fuertemente pigmentada en los 360 grados (figura 2).

Figura 2. Gonioscopía.

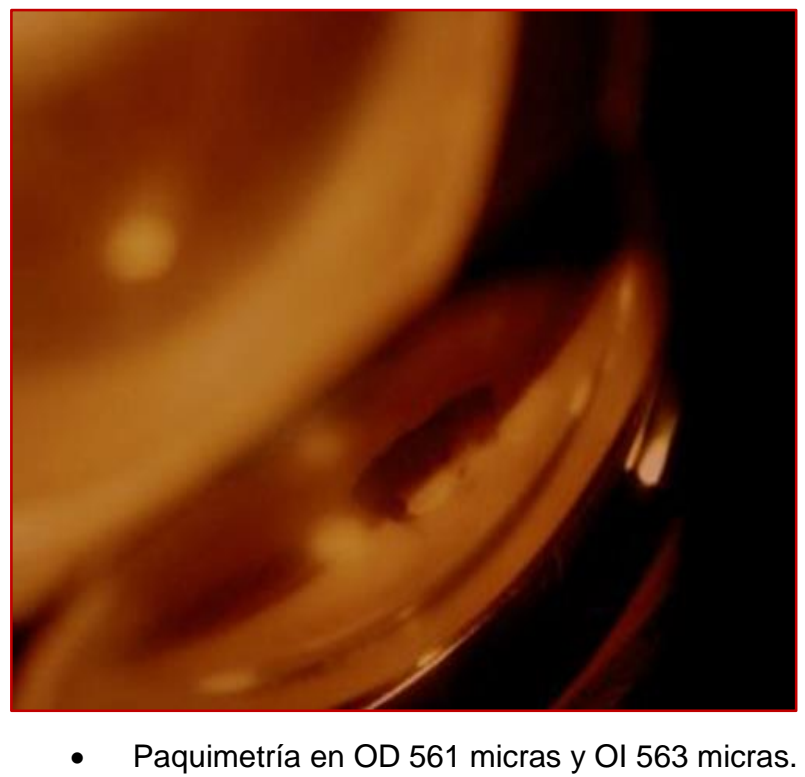

La biomicroscopía ultrasónica confirmó la presencia de la masa iridiana, su localización en la periferia del iris, con extensión al ángulo de la cámara anterior con unas dimensiones de $5 \times 3 \mathrm{~mm}$ de base y $2 \mathrm{~mm}$ de altura. En el fondo del Ol se observó que la papila estaba completamente excavada y pálida dejando ver la lámina cribosa.

Se indicó tratamiento con dorzolamida más timolol en colirio una gota cada 12 horas y valorar dentro de 15 días. Al cabo de este tiempo la tensión ocular del OD era de $13.5 \mathrm{mmHg}$ y en OI $35 \mathrm{~mm}$ de $\mathrm{mmHg}$, para confirmar el diagnóstico se le realiza biopsia escisional de la masa (irido-resección).

En el estudio anatomopatológico se observó una proliferación de células névicas pleomórficas que reemplazaban el estroma normal del iris. Se le realizaron pruebas de extensión, que resultaron negativas para enfermedad sistémica. Debido a la naturaleza maligna de la lesión se decidió completar el tratamiento con braquiterapia epiescleral adyuvante con iodo-125. La hipertensión resultante fue controlada con tratamiento farmacológico. En la actualidad, no se ha observado recidiva local ni extensión metastásica de la enfermedad. La tensión ocular se mantiene estable contratamiento farmacológico.

\section{Discusión}

La presencia de una lesión pigmentada de aparición súbita hace plantearse el diagnóstico diferencial entre tumores pigmentados benignos, como nevus, lesiones quísticas y tumores malignos como el melanoma, ya que éste es el tumor más frecuente en esta localización ${ }^{1,4,5}$.

Los melanomas de úvea suelen presentarse en la cuarta, quinta, sexta y séptima década de la vida. Pueden ser asintomáticos y se diagnostican por casualidad en un examen de rutina con el oftalmoscopio, o pueden presentarse como una disminución lenta y progresiva de la visión o con hipertensión ocular. Estas características coinciden con los datos generales de la paciente que se describe, la que acudió por pérdida lenta de su visión sin otra sintomatología, lo cual alerta sobre la necesidad de realizar un examen minucioso hasta encontrar la causa de la pérdida visual. El estudio por biomicroscopia ultrasónica 
es de gran importancia para la clasificación de estos casos y para la decisión de una conducta terapéutica. La mayoría de los pacientes que presentan melanoma uveal, la búsqueda inicial de una posible afectación sistémica al momento del diagnóstico no arroja resultados positivos, sin embargo, es el fenómeno que ocurrió en la paciente; donde a pesar del tamaño de la lesión y el tipo histológico del mismo, no existió evidencia hasta la fecha de la presencia de recidivas o metástasis ${ }^{6,7,8}$.

La biopsia escisional permite tratar la lesión y hacer un diagnóstico anatomopatológico de certeza. La dificultad para obtener márgenes quirúrgicos amplios que garanticen ausencia de recurrencia hace fundamental la asociación de braquiterapia epiescleral como tratamiento adyuvante ${ }^{9,10,11}$.

\section{Conclusiones}

En pacientes que presentan una clínica de glaucoma unilateral refractario a tratamiento farmacológico junto con dispersión de pigmento es importante una exploración exhaustiva destacando el papel de la biomicroscopía ultrasónica. En los pacientes con melanoma de iris localizado, la resección junto con braquiterapia adyuvante es efectiva en el control de la enfermedad. Es por esto por lo que se debe tener en cuenta como diagnóstico diferencial para realizar una detección temprana y dar un tratamiento oportuno para evitar posibles complicaciones en el paciente.

\section{Conflicto de intereses}

Ninguno declarado por los autores.

\section{Referencias}

1. Gallego Y, Mendicute J, Ruiz M, Ruiz I, Ubeda M. Melanocitoma de cuerpo ciliar. Arch Soc Esp Oftalmol [en línea]. febrero de 2005 [citado 8 de enero de 2017];80(2):109-12. Disponible en: http://scielo.isciii.es/scielo.php?script=sci_abstract\&pid=S0365$66912005000200010 \&$ Ing =es\&nrm=iso\&tlng=es

2. Bianciotto C, Saornil MA, Muiños Y, Méndez MC, Blanco G, FrutosBaraja JM, et al. Hipertensión ocular como principal forma de presentación del melanoma uveal. Arch Soc Esp Oftalmol [en línea]. enero de 2005 [citado 18 de enero de 2017];80(1):27-33. Disponible en: http://scielo.isciii.es/scielo.php?script=sci_abstract\&pid=S0365$66912005000100006 \&$ Ing $=$ es\&nrm=iso\&tlng=pt
3. Ajamil Rodanés S, García-Álvarez C, Saornil Álvarez MA, López-Lara $\mathrm{F}$, Frutos Baraja JM, Quiñones $\mathrm{M}$. Glaucoma melanomalítico secundario a melanoma de iris. Arch Soc Esp Oftalmol [en línea]. 2017 [citado 7 de enero de 2017];92(8):379-81. Disponible en: https://medes.com/publication/123022

4. Pérez García D, del Castillo Carrillo C, Alemañy Rubio E, Rodríguez Rodríguez V, Rúa Martínez R, Hernández Cedeño C. Metástasis coroidea de un carcinoma de mama. Rev Cuba Oftalmol [en línea]. 2012 [citado 11 de febrero de 2017];25(1):161-168. Disponible en: http://scielo.sld.cu/scielo.php?script=sci_arttext\&pid=S086421762012000100018

5. Aja LT, Suárez OF. Melanoma de coroides con metástasis axilar. Presentación de un caso Choroidal Melanoma with Axillary Metastasis. A Case Report. [citado 11 de febrero de 2017]; Disponible en: http://www.medigraphic.com/pdfs/finlay/fi2013/fi134f.pdf

6. Rodríguez Pargas A, Gallardo Roca L, Chávez Pardo I, Borrego Lastre X. Melanoma de coroides: presentación de un caso. Rev Arch Méd Camagüey [en línea]. 2012 [citado 8 de enero de 2017];16(3):310-317. Disponible

http://scielo.sld.cu/scielo.php?pid=S1025-

02552012000300008\&script=sci_arttext\&tIng=en

7. Fernández Soler JA, Fuentes Hidalgo JE, Orges Ramírez M, Martínez Quintana S, Serrano Fuentes T. Melanoma maligno de coroides: a propósito de un caso. Correo Científico Méd [en línea]. 2017 [citado 8 de enero de 2017];21(1):295-304. Disponible en: http://scielo.sld.cu/scielo.php?pid=S1560-

43812017000100026\&script=sci_arttext\&tlng=pt

8. Damato EM, Damato BE. Detection and Time to Treatment of Uveal Melanoma in the United Kingdom: An Evaluation of 2384 Patients. Ophthalmology [en línea]. 1 de agosto de 2012;119(8):1582-9. Disponible

http://www.sciencedirect.com/science/article/pii/S016164201200109 1

9. Chang MY, McCannel CA, McCannel TA. Untreated iris melanoma complicated by hyphema and uncontrolled glaucoma responsive to iodine-125 brachytherapy. Retin Cases Brief Rep [en línea]. 2016 [citado 12 de febrero de 2017];10(1):44-47. Disponible en: http://journals.Iww.com/retinalcases/Abstract/2016/01010/UNTREAT ED_IRIS_MELANOMA_COMPLICATED_BY_HYPHEMA_AND.11.a spx

10. Shields CL, Shah SU, Bianciotto CG, Emrich J, Komarnicky L, Shields JA. Iris Melanoma Management with lodine-125 Plaque Radiotherapy in 144 Patients: Impact of Melanoma-Related Glaucoma on Outcomes. Ophthalmology [en línea]. 1 de enero de 2013;120(1):55-61. Disponible en: http://www.sciencedirect.com/science/article/pii/S016164201200613 6

11. Shields JA, Shields CL. Management of Posterior Uveal Melanoma: Past, Present, and Future. Ophthalmology [en línea]. 1 de febrero de 2015;122(2):414-28. Disponible en http://www.sciencedirect.com/science/article/pii/S016164201400819 7

Enferm Inv. (Ambato) 2(3):115-117 